\title{
COHESIÓN TERRITORIAL Y SOSTENIBILIDAD EN LOS VALLES DEL BIERZO
}

\author{
Territorial Cohesion and Sustainability in the Bierzo Valleys \\ Coesão territorial e sustentabilidade nos Vale do Bierzo
}

\author{
Carlos MONTES PÉREZ \\ Universidad de Salamanca \\ cmontes@usal.es \\ ORCID: https://orcid.org/0000-0002-7619-3406
}

Fecha de recepción: 3/01/2021

Fecha de aceptación: 13/01/2021

RESUMEN: El artículo presenta las conclusiones de un trabajo etnográfico llevado a cabo durante los últimos cinco años en la localidad de Balboa, centro de uno de los valles leoneses cercanos a la frontera con Galicia. El estudio se centra en esta localidad por la peculiaridad que presenta en relación a otras localidades cercanas, también de zona de montaña, que se han ido quedando sin población en los últimos años. A diferencias de estas zonas despobladas, en Balboa, debido a la confluencia de varios factores, la población se ha mantenido en un ejercicio meritorio de resiliencia rural. Pero, a su vez, sus habitantes y sus dirigentes políticos han logrado colocar a Balboa en el mapa de los lugares que ofrecen un determinado tipo de ocio que ha hecho que sean numerosos los visitantes que a lo largo de los últimos años han pasado por la localidad. Este fenómeno da forma a lo que describimos como una ruralidad fluida donde lo importante ya no es tanto la residencia, sino la idea de flujo.

Palabras clave: Bierzo; sostenibilidad; ruralidad fluída; desarrollo rural; ruralidad de montaña. 
CARLOS MONTES PÉREZ

COHESIÓN TERRITORIAL Y SOSTENIBILIDAD EN LOS VALLES DEL BIERZO

ABSTRACT: The article presents the conclusions of an ethnographic work carried out during the last five years in the town of Balboa, the center of one of the valleys of Leon near the border with Galicia. The study focuses on this town because of the peculiarity it presents in relation to other nearby towns, also in the mountain area, which have been running out of population in recent years. Unlike these unpopulated areas, in Balboa, due to the confluence of various factors, the population has remained in a meritorious exercise of rural resilience. But, in turn, its inhabitants and its political leaders have managed to place Balboa on the map of the different types of a certain type of leisure that has led to numerous visitors who have passed through the town in recent years, giving shape to what we describe as a fluid rurality where the important thing is no longer so much the residence, but the idea of flow.

Key words: Bierzo; sustainability; fluid rurality; rural development; mountain rurality.

RESUMO: O artigo apresenta as conclusões de um trabalho etnográfico realizado durante os últimos cinco anos na localidade de Balboa, centro de um dos vales de Leão, perto da fronteira com a Galiza. O estudo centra-se nesta localidade pela peculiaridade que apresenta em relação a outras localidades vizinhas, também na zona serrana, que têm vindo a ficar sem população nos últimos anos. Ao contrário destas áreas despovoadas, no Balboa, devido à confluência de vários factores, a população manteve-se num meritório exercício de resiliência rural. Mas, por sua vez, seus habitantes e seus líderes políticos conseguiram colocar Balboa no mapa das diferentes modalidades de um determinado lazer que tem levado a numerosos visitantes que passaram pela cidade nos últimos anos, dando forma ao que descrevemos como uma ruralidade fluida onde o importante não é tanto a residência, mas a ideia de fluxo.

Palavras chave: Bierzo; sustentabilidade; ruralidade fluida; desenvolvimento Rural; ruralidade montanhosa.

\section{INTRODUCCIÓN}

En el mes de febrero del año 2018 una de las inmobiliarias más exclusivas de España, con sede en Barcelona ponía a la venta la última mina de la comarca del Bierzo, en la provincia de León, en el Noroeste de España. Este terreno de más de 8.000 hectáreas permite, además del anunciado aprovechamiento extractivo, otros fines como el cinegético, el industrial, el maderero o el turístico. La puesta a la venta de esta antigua explotación es la última gota de un lento y contínuo cierre de industrias extractivas en la zona y confirma la profunda crisis que ha terminado con el sector minero del carbón. Un modo de producción, adoptado desde hace casi un siglo en los valles montañosos del norte de España que ha transformado, de un modo muy significativo el mundo rural, bien sea de algunas comarcas 
CARLOS MONTES PÉREZ

COHESIÓN TERRITORIAL Y SOSTENIBILIDAD EN LOS VALLES DEL BIERZO

mineras leonesas, asturianas, como también de otras partes del territorio nacional. Las actividades agrícolas y ganaderas que durante largo tiempo fueron las actividades centrales en estos territorios fueron desplazadas a un ámbito secundario y complementario en la mayor parte de las localidades, mientras que, en otras muchas, fueron directamente abandonadas. La actividad extractiva, la minería se convirtió en el sector que impulsaba, casi al ritmo de un galope, la economía rural de la comarca y fue, por esto, mayoritariamente bienvenido.

A pesar de los múltiples problemas medioambientales y de salud que conllevan, ha generado trabajo y producido riqueza y bienestar durante los últimos setenta años. Pero este modelo productivo ha llegado a su fin. Son numerosos los pueblos en la comarca que se han resistido a este final, pero parece que no hay vuelta atrás. El desmantelamiento de las numerosas minas y empresas de la comarca ha ido dejando ya señales de pobreza, desempleo, despoblación, desigualdad, desilusión y un enorme aumento de falta de cohesión social y territorial entre distintas comarcas y localidades.

Desde el desarrollo de este modelo productivo en la comarca del Bierzo, los municipios rurales han quedado fuertemente divididos entre aquellos que formaron parte de los distintos cotos mineros, ya sean en su extracción de carbón, hierro, wolframio o cualquier otro mineral, y aquellos que, por su situación geográfica y por la composición de sus suelos no pudieron integrarse y formar parte de esta potente, pero a la larga insostenible industria. Estas localidades rurales no extractivas se sitúan en el llamado Bierzo bajo, zona geográfica que ocupa el centro de la hoya berciana. Muchos de estos municipios han mantenido una destacada y creciente producción agrícola basada en productos de gran calidad con denominación de origen, entre los que destacan el pimiento, la manzana y la pera, entre otros. Junto a esto, y también de un modo muy creciente y prometedor ha ido gestándose una importante industria vitivinícola en esta parte no extractiva del territorio. De la tradición doméstica se ha pasado en unas décadas a una potente industria agroalimentaria exportadora de calidad que se ha convertido en uno de los principales motores de la comarca. (González, 1984; Alonso, 2003; Cortizo, 1992).

Pero esta perspectiva rural no se agota en esta descripción, aún presenta, en este territorio tan característico, un conjunto de municipios que han quedado descolgados de estas dos formas productivas expuestas anteriormente. No han sido relevantes para la industria extractiva y además, su condición de pueblos de montaña con una dura orografía y climatología no les ha permitido desarrollar una agroindustria que haga sostenible su nivel de vida. Son localidades que, a pesar de encontrarse en entornos naturales privilegiados, se han ido despoblando y quedando olvidados, desconectados, necesitados de una profunda renovación que los cohesione y los vincule de nuevo social y territorialmente con otros territorios que contribuyan a su sostenibilidad.

Son, por tanto, estos territorios el objeto de nuestra investigación, ya que no cumplen con aquellos elementos que determinan la deseada cohesión territorial. 
Ahora bien, ante este desiderátum, cabe reflexionar inicialmente sobre algunos interrogantes, cómo son: ¿qué factores muestran esta falta de cohesión?, ¿cómo podríamos delimitar objetivamente esta situación?, ¿qué elementos han de tenerse en cuenta para mejorar su condición actual?

Estos territorios de montaña no cumplen, por tanto, con aquellos elementos que determinan la cohesión territorial y han de ser objeto de atención y estudio, tal y como propone la Comisión Europea en su Libro Verde sobre esta temática. En primer lugar, estos territorios muestran un desarrollo socioeconómico desequilibrado. Aparecen significativas desigualdades de renta, de actividad productiva, de empleo entre distintas localidades y entre estas zonas montañosas y las localidades mineras y del bajo Bierzo. También es reseñable el grado inadecuado de articulación de estos municipios a través de las redes de transporte y de infraestructuras. Así mismo resulta complicado encontrar condiciones semejantes de accesibilidad a los servicios públicos, a las infraestructuras y de los equipamientos. No cabe duda de que la falta de consecución y de logro de esta cohesión territorial lleva aparejada una lenta y agónica despoblación con la consiguiente pérdida general que esto conlleva (CEE, 2008).

Algunos territorios se resignan a esta situación y sus habitantes, sus dirigentes políticos y referentes sociales han arrojado ya la toalla y solamente esperan de forma agónica la desaparición del municipio cuando emigre el último habitante o se cierre la última casa. En cambio, otros territorios han plantado cara a la situación en la que se encuentran, lo hicieron ya a través de las políticas de desarrollo rural, pero, en muchos casos no ha tenido el efecto esperado. Sin embargo, lo siguen intentando, sus gentes no se resignan.

Nuestra investigación aborda de forma etnográfica uno de estos casos, de los numerosos que se extienden por el mundo rural, teniendo en cuenta, sobre todo, la diversidad de sus propuestas y sus efectos. El lugar referido, por tanto, es el valle de Balboa, en la zona oeste de la comarca del Bierzo, dentro de la provincia de León, muy cercano al límite con la comunidad autónoma de Galicia. Este valle, tradicionalmente dedicado a la agricultura y a la ganadería ha experimentado a lo largo de las últimas décadas un pronunciado descenso de la población, y un deterioro y abandono de su patrimonio tanto natural como cultural, a pesar de la riqueza y la belleza de sus paisajes, tal y como pone de manifiesto este pasaje del escritor José María Cuadrado en 1855 en su obra "Recuerdos y bellezas de España" El texto presenta la comarca estudiada del siguiente modo:

Vamos a entrar en un país encantado, de nombre y fisonomía y producciones peculiares respecto de la provincia que lo contiene; circunscrito por ásperas e imponentes sierras, rico en metales, exuberante en aguas, copioso y variado en frutos, pintoresco en sus perspectivas, poético en sus tradiciones, poblado de monasterios y castillos, fecundo en antiguas memorias y preciosos monumentos. Explotáronlo cual aurífero minero los romanos, dejando en él vestigios indelebles de su grandeza 
CARLOS MONTES PÉREZ

COHESIÓN TERRITORIAL Y SOSTENIBILIDAD EN LOS VALLES DEL BIERZO

y perseverancia; convirtióse durante la monarquía goda en austera Tebaida, que asolada momentáneamente por avenidas de sarracenos, refloreció poco después con nuevos ejemplos de santidad; y bajo el paternal dominio de los abades y la protectora espada de los caballeros, agrupáronse sus ideas, crecieron sus villas, desmontáronse sus selvas y baldíos, y transformáronse en vergeles sus valles y cañadas.

\section{El VAlLe de Balboa. Localización}

El valle de Balboa es el valle más pequeño y meridional de los valles de Ancares, en la comarca del Bierzo, al oeste de la provincia de León. Presenta una extensión de algo más de cincuenta kilómetros cuadrados y se encuentra bañado por las aguas del río que le da nombre: el río Balboa. La altitud media del valle ronda los $950 \mathrm{~m}$. Destaca en esta zona su enorme riqueza natural y sus bellos paisajes, así como algunos restos arquitectónicos de diferentes épocas como la interesante iglesia románica de Santa Marina, el Castillo de Balboa y algunas pallozas, a saber, viviendas tradicionales que actualmente han sido recuperadas con un claro interés turístico, pero que ha formado parte del territorio dotándolo de una identidad propia, vinculada al llamado noroeste ibérico, que enlaza con Galicia y con Asturias. El valle se compone de numerosas pequeñas localidades rodeadas todas ellas de verdes prados y de grandes conjuntos de castaños, que en el territorio adoptan el nombre de "sotos". Destaca como lugar central del valle y referencia administrativa, la localidad de Balboa, la más importante y centro de nuestra investigación. Eje de la vida del valle, y lugar que recoge los principales servicios y actividades turísticas y de ocio que en él se desarrollan. Junto a Balboa, Pumarín y Cantejeira son lugares que cuentan también con edificaciones de pallozas y concentran también algunos servicios. El valle además se compone de otras localidades como son: Castañeiras, Vilariños, Chan de Villar, Quintela, Ruidelamas, Ruideferros, Castañoso, Valverde, Villanueva, Fuente de oliva, Villafeile, Lamagrande, Villariños, Pumarin, Villarmarín o Parajis. La agricultura y, sobre todo, la ganadería, han formado parte de la vida del valle desde tiempos que se pierden en la memoria. Algunos de estos municipios han tenido en los últimos tiempos una presencia destacada en los medios de comunicación por su deseo de unirse a la comunidad gallega y dejar de pertenecer a la macrocomunidad de Castilla y León.

Este deseo y esta aspiración canalizada por alguno de los alcaldes pedáneos no es baladí, ni responde únicamente a un principio de oportunidad, la especial situación del valle cercano al límite con la Comunidad Autónoma de Galicia ha contribuido a que sea uno de los municipios de la provincia de León, y de la Comunidad Autónoma de Castilla y León más fuertemente galleguizado, no solo en el habla habitual, sino sobre todo también en sus topónimos, modos de construcción, de creencias y formas de vida. 


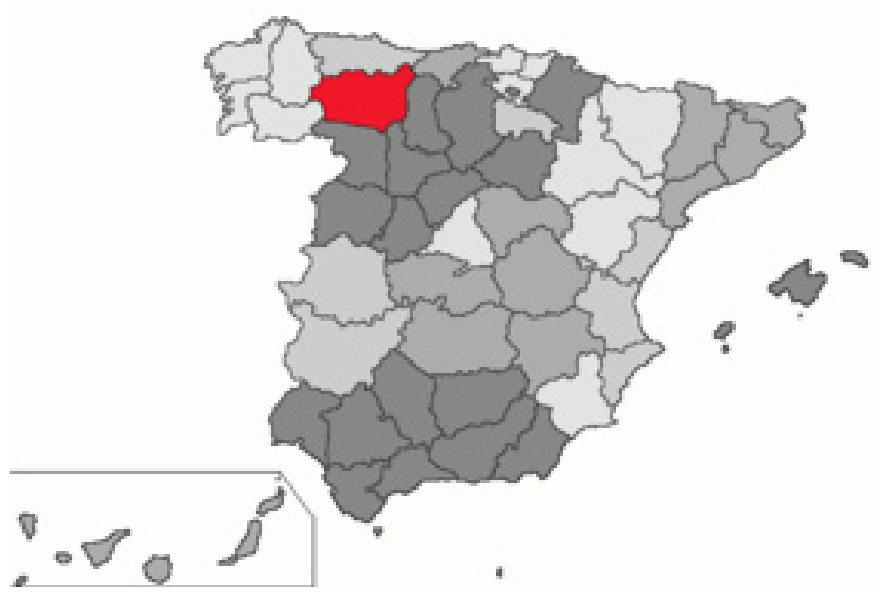

Figura n. ${ }^{\circ}$ 1. Localización de la provincia de León en el Mapa de España.

Según el profesor Jesús García García (1994) las referencias leonesas que se encuentran en el valle son muy modernas. Las bondades de su situación natural quedan reflejadas en el nombre que originariamente proviene del latín VallisBona, es decir, el buen valle. El adjetivo «bona» en gallego se transformó en «boa» y así quedó este territorio definido como «valboa». El cambio de la «V» inicial por la "b» actual que se ha mantenido hasta ahora en el nombre nos resulta desconocido, no ha sido analizado de forma satisfactoria por los lingüistas que han abordado la toponimia del lugar, y está sometido a variadas interpretaciones.

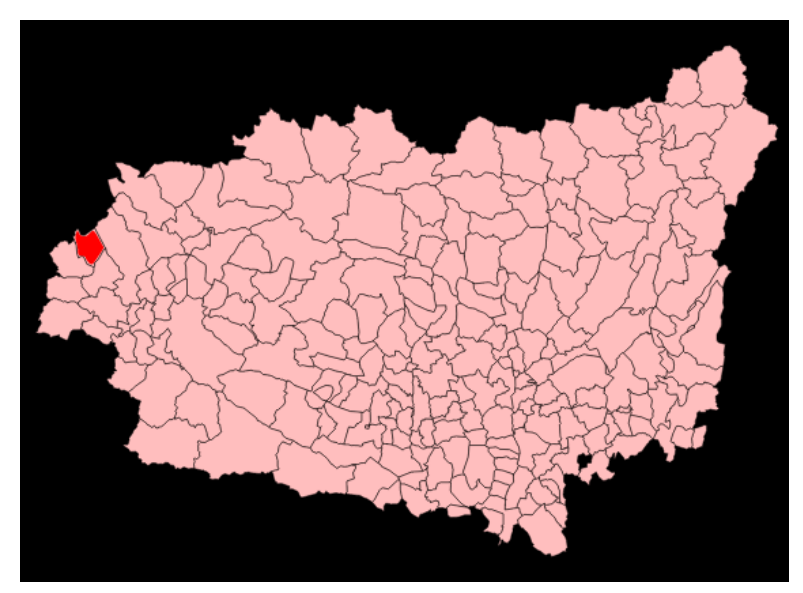

Figura n. ${ }^{\circ}$ 2. Localización de Balboa en el extremo occidental de la provincia de León. 


\section{SUS GENTES}

Como resulta habitual en el análisis de las zonas rurales en contexto de montaña o alejados de los núcleos rurales, uno de los efectos más llamativos de la desconexión territorial es la despoblación. Este es un fenómeno que ha llamado la atención de numerosos científicos sociales que han puesto el foco en distintos lugares de la geografía española desde hace varios años (García Sanz, 1996; Saéz, 2016).

La densidad de población se encuentra alrededor de 8 hab. $/ \mathrm{km}^{2}$, lo que sitúa a este territorio en la categoría de área escasamente poblada. Pasear por todos estos lugares, más allá del mero dato estadístico provoca un escalofrío al percibir lo que significa un verdadero desierto demográfico. Si observamos con detenimiento la gráfica del histórico de población desde principios de siglo pasado podremos apreciar que el número de habitantes del valle nunca ha sido muy numeroso. Ahora bien, sí que podríamos afirmar que se ha mantenido un cierto equilibrio demográfico y social. Su población se ha encontrado estabilizada y se ha conseguido una forma económica tradicional sostenible a través de una especial diversificación entre la producción agrícola y la explotación ganadera en tiempos pretéritos. Esto es claramente perceptible desde el principio del siglo XX hasta la década de los años 30. En el caso que nos ocupa, el valle de Balboa, este equilibrio se va alterando más allá de esta fecha, la caída de la población es constante, aunque lenta a partir de los años 40 hasta los años 70. Y, a partir de ese momento el declive poblacional es desolador. En los últimos cincuenta años el valle ha perdido el $75 \%$ de su población.

Ahora bien, si ajustamos un poco más la mirada y nos centramos en las últimas dos décadas podemos ver cómo su población se ha estabilizado, no ha habido descensos bruscos, como en otros lugares parecidos. Incluso apreciamos una leve subida poblacional en el año 2006, lo cual no es muy frecuente en entornos de montaña y en el contexto de crisis productiva que hemos descrito. Este mantemiento de la población no es fruto del azar histórico, sino más bien, es el resultado de un conjunto de acciones llevadas a cabo desde distintas entidades y personas que se han conjurado para tratar de dotar de sostenibilidad a un modo de vida plenamente anclado en la tradición pero que aspira a insertarse en la modernidad globalizada sin renunciar al paisaje, y al modo de vida deseado. Este es, sin duda, el reto de una gran mayoría de los territorios rurales y de montaña de nuestro país. Poner de manifiesto estos esfuerzos y las estrategias seguidas a través de ellos para que obtengan visibilidad y puedan ser ejemplo en otros lugares es el objetivo principal de este trabajo etnográfico que se inserta dentro de los esfuerzos de la cátedra de territorios rurales y desarrollo sostenible que se asienta en la sede del Centro Asociado de la Uned en Ponferrada. 


\section{Balboa (Municipio) - Evolucion del numero de Habitantes}

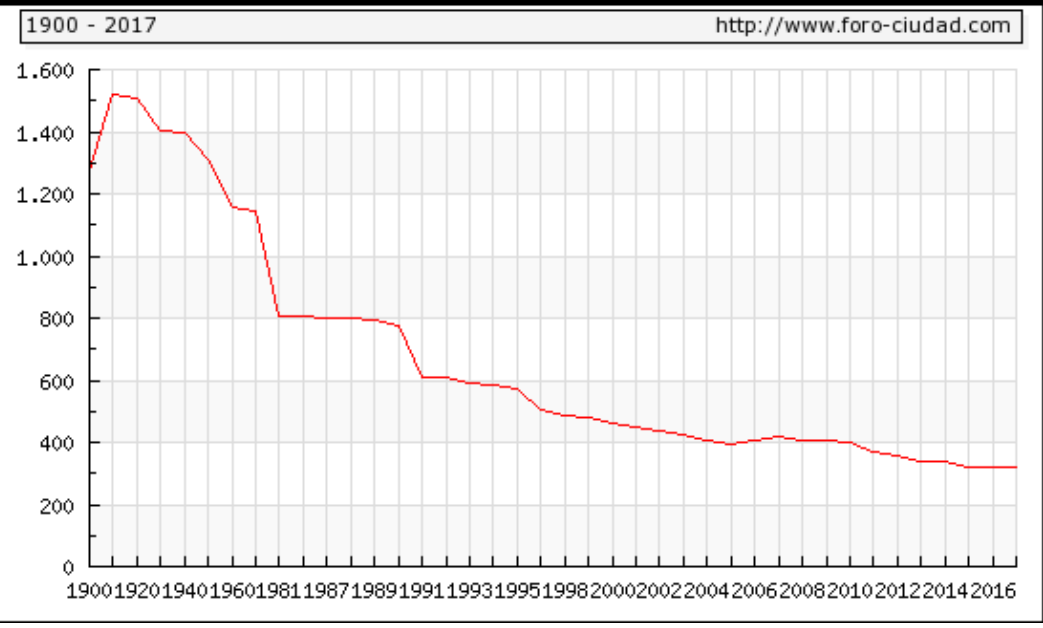

Figura n. ${ }^{\circ}$ 3. Gráfico de población desde el año 1900 hasta el año 2015.

\section{Balboa - Habitantes segun Lugar de nacimiento - 2017}

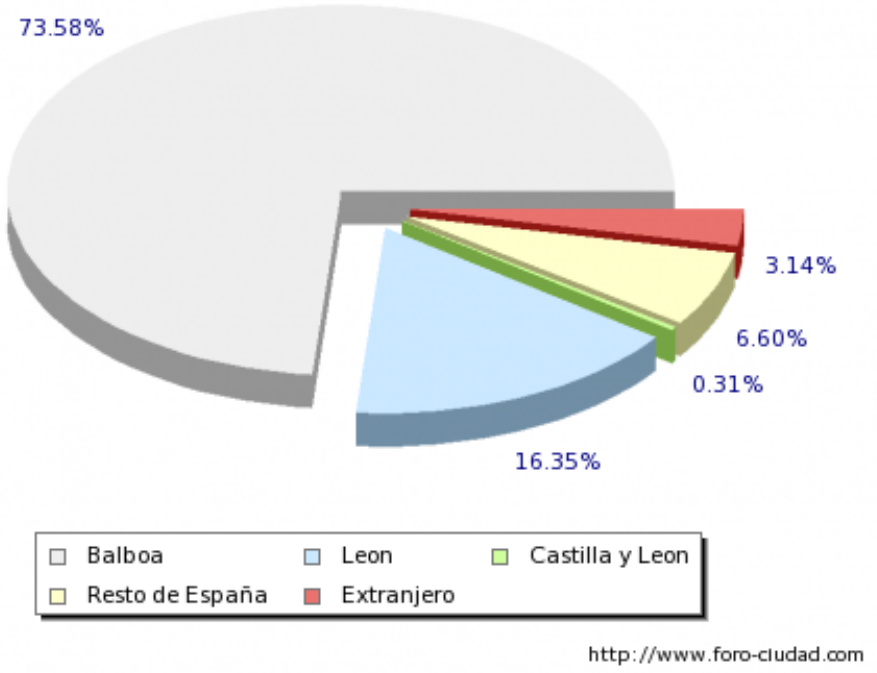

Figura n. ${ }^{\circ}$ 4. Habitantes según su procedencia. 
CARLOS MONTES PÉREZ

COHESIÓN TERRITORIAL Y SOSTENIBILIDAD EN LOS VALLES DEL BIERZO

\section{COHESIÓN TERRITORIAL: HACIA UNA INTEGRACIÓN MÁS Y SOSTENIBLE DE LOS ESPACIOS RURALES}

¿Por qué es necesario un replanteamiento de la situación de los espacios rurales? La pregunta en este momento no puede ser más pertinente. Estamos en un momento de cambio en la clásica relación entre lo rural y lo urbano. Aún, no tenemos la claridad suficiente para valorar lo propuesto, pero, sin duda, la idea de la necesidad de una mejor cohesión territorial es una posibilidad de encontrar un equilibrio en este histórico y complicado dualismo. En primer lugar, la idea de ruralidad y la condición de espacio rural en nuestro país se ha diversificado enormemente. La comarca del Bierzo, como hemos señalado anteriormente es un buen ejemplo de ello. La situación está muy lejos de ser homogénea. Por el contrario, se encuentra claramente marcada por una heterogeneidad interna que nos condiciona a analizar casi cada espacio rural de forma independiente. Aún siendo notable la presencia en ellos de la actividad agraria, sus distintas formas de explotación, sus rendimientos y productividades, sus modos de gestión, así como sus experiencias de modernización y de intensificación varían sensiblemente. Basta con observar detenidamente la realidad del mundo rural español para percibir el significativo contraste entre territorios, no sólo desde el punto de vista agrícola, sino también desde la perspectiva social, cultural, ambiental, de ocio y paisajística. En este sentido, y sin ánimo de exhaustividad, podríamos ofrecer una tipología de áreas rurales en las que se distinguieran aquellas que cuentan con un buen nivel de desarrollo basado en la agricultura y que cuentan con sistemas en general bien articulados. Estos espacios cuentan con áreas bien dotadas de infraestructuras y equipamientos sociales que se asemejan mucho a los conseguidos en el ámbito urbano, y que incluso han ganado población. Dentro de estos territorios pueden encontrarse movimientos tanto cooperativos como empresariales eficientes desde el punto de vista del mercado que se constituyen como verdaderos motores de la economía local. Este es el caso de la industria agroalimentaria en la comarca del Bierzo, representado sobre todo por la pujanza del sector vitivinícola. Sin duda, estos espacios presentan un modelo de ruralidad dinámico que ha sido objeto ya de múltiples y variados estudios.

En cambio, en el otro extremo nos encontramos con áreas en zonas de interior o de montaña cuya ruralidad es radicalmente diferente. Presentan importantes déficits estructurales y fuertes tensiones demográficas que han puesto en cuestión el cumplimiento de alguno de los objetivos del llamado desarrollo rural. Como analizaremos en el caso que nos ocupa, estos espacios suelen estar alejados de los centros urbanos, están acostumbrados a sufrir importantes déficits estructurales en servicios considerados básicos, tener bajos niveles de población que dificulta cualquier iniciativa que necesite un mercado cercano y de proximidad. Uno de los problemas fundamentales que estos espacios han de abordar es cómo romper el umbral de población mínimo que se necesita para hacer posible cualquier proyecto de desarrollo y justificar, desde el punto de vista social, las inversiones y 
equipamientos que serían necesarios para poder garantizar la calidad de vida a los habitantes que decidieron quedarse allí. Este tal vez ha sido uno de los problemas fundamentales con los proyectos llevados a cabo dentro de las políticas del llamado desarrollo rural. Se ha invertido en infraestructuras y equipamientos demandados desde estas áreas rurales, pero no hay población suficiente para utilizarlos, aprovecharlos y que resulten eficientes. Algunos de ellos han contribuido a la sensación de abandono y de soledad que se percibe en muchos espacios rurales. El reto, desde nuestra perspectiva y cómo veremos en nuestro trabajo, consistirá en primer lugar en revalorizar estas áreas rurales desde perspectivas que vayan más allá de su mero sentido productivo.

Conviene no olvidar el importante paso dado en la consideración de estos territorios como un valor intangible, incluso fuera del mercado y vinculado a la preservación y disfrute de paisaje o la mera conservación del espacio natural. De forma lenta pero constante estamos asistiendo a un cambio de significado del viejo concepto de "mundo rural». Cada vez tiene más peso este valor ambiental que se encuentra vinculado a actividades de larga tradición histórica (actividades agropastoriles, cinegéticas, micológicas, apícolas, pesqueras, etc.) Renunciar definitivamente a estos espacios es renunciar a nuestra historia, conllevaría la pérdida de elementos fundamentales de biodiversidad social y cultural vinculados con las prácticas locales, lo que supondría de facto renunciar a nuestra identidad. Pero, solamente tener esto en cuenta convertiría a nuestro mundo rural más desestructurado en un museo convirtiendo, por tanto, a sus habitantes en piezas del mismo, al estilo del viejo debate sobre la relación del mundo contemporáneo con las poblaciones indígenas. (Amselle, 2010).

El abandono total aumenta el riesgo de deterioro general ambiental como se puede ver en el número creciente de incendios forestales.

Aquí radica principalmente el reto para el futuro de estos espacios. ¿Cómo garantizarle a la población unas condiciones dignas de vida?, pero también, ¿cómo hacer atractivos estos espacios para que no se abandonen y para que incluso aumenten su población? El reto no es menor. Las pruebas para ello se encuentran objetivamente visibles en la cantidad de dinero invertido desde hace décadas en políticas públicas tanto nacionales como internacionales para evitar la despoblación sin que el resultado haya sido efectivo hasta el momento presente. (Esparcia, 2004).

La esperanza, a nuestro juicio, pasa por conseguir conectar estos espacios rurales a través de formas diversas e imaginativas, tal y como vamos a presentar en la segunda parte de nuestro trabajo. Se trata, por tanto, de entender el territorio, la habitabilidad del mismo y el enraizamiento en él de una forma distinta, más fluida, con una mejor regulación donde lo que esté presente también sea la idea de flujo. De una manera acertadísima el profesor Gómez Pellón ha acuñado el término de «ruralidad líquida» para describir este nuevo proceso (Gómez, 2012) Por tal motivo destacaremos en nuestro trabajo dos conceptos claves de esta nueva ruralidad líquida: la conectividad y la idea de flujo. 
Algunos estudiosos del mundo rural olvidan, a veces, que los avances en la tecnología sirven para conectar a los seres humanos en la actualidad como nunca se había hecho antes. Este nuevo mundo digital abre nuevas vías que permiten abordar la viabilidad de estas áreas rurales de otra manera ya que permitiría establecer servicios de proximidad y de consulta con relativa facilidad. Especialmente significativo puede ser el caso de la salud, ya que muchos de los habitantes del mundo rural de montaña sienten miedo ante la posibilidad de la enfermedad y no contar con la cercanía de un conocimiento experto. Las nuevas tecnologías permiten ya, con inversión y voluntad política la ruptura de esta desconfianza, con la consiguiente seguridad y mejora de la calidad de vida.

Esta viabilidad apoyada en las nuevas tecnologías permite desarrollar actividades de teletrabajo, así como también de formación y de apertura de mercados a un mundo que de forma creciente demanda más productos ecológicos y más sanos. En definitiva, esto propicia la reducción de su tradicional aislamiento y abre, por tanto, la posibilidad de una mayor conexión o cohesión territorial. A través de las comunicaciones en redes se ha modificado de un modo muy sustantivo la idea del espacio y de la casa como lugar cerrado. Ahora ya cada pantalla de ordenador conectada es una ventana al mundo, del mismo modo que el mundo se cuela a través de cada ventana. Es lo que Remedios Zafra ha descrito con maestría como la "casa hiperconectada". (Zafra, 2010).

Ahora bien, no solo se puede mejorar la conectividad a través de las nuevas tecnologías. De hecho, hay múltiples estudios que señalan cómo el creciente atractivo de los territorios rurales como lugares de segunda residencia para todo un conjunto variado de población que los elige como lugares no permanentes sino estacionales ha modificado la vida rural y ha activado incluso áreas rurales absolutamente abandonadas y ha abierto posibilidades nuevas de consumo en los espacios rurales (Murdoch y Pratt, 1997). Al mismo tiempo se percibe el cambio en la valoración de lo rural en aquellos que fijan por primera vez su residencia en áreas rurales procedentes de anteriores experiencias y modos de vida en entornos urbanos y que conocemos bajo el calificativo de «neoruralidad». Estos nuevos modelos de vida constituyen una forma interesante de reactivar algunas áreas despobladas y desprotegidas, pero están lejos de constituir una solución definitiva. Tanto la presencia de segundas residencias activas, como el aumento del turismo rural, y la presencia de la neoruralidad son elementos, sin duda, que ayudan a la superación del aislamiento rural y estimulan la conectividad, la recién mencionada "Cohesión territorial". Ahora bien, como la realidad se encarga constantemente de mostrarnos, el asunto es realmente complejo, y en algunos territorios se debería

1. Si bien en la mayoría de los países latinoamericanos, la denominación de neoruralidad remite no solo a este fenómeno de nuevos habitantes del espacio rural, sino que también abarca nuevas formas productivas a las tradicionales, así como una diversificación en los sistemas económicos tradicionales. 
abordar como una verdadera cuestión de Estado. La Unión Europea ya ha dado algunos pasos importantes en este sentido, y nos consta que el gobierno también se encuentra en la misma dirección para implicar a todas las instituciones que, de un modo u otro, intervengan en el mundo rural. Dadas las características mostradas de estos territorios, así como la urgente necesidad de impulsar en ellos la más amplia cooperación social e institucional, es muy importante que cuenten en su gobernanza con las instituciones locales, ya que, a pesar de que sus medios son siempre limitados, la capacidad de imaginación y el conocimiento de su territorio y de su potencialidad son, en gran medida, ilimitados. Grupos de acción local, organizaciones agrarias, asociaciones en defensa del medio ambiente, cooperativas locales, emprendedores locales, y ayuntamientos son esenciales para este sistema de gobernanza. Resultan esenciales para la generación de confianza y para evitar el desaliento, y funcionan como un gran capital social para reactivar la identidad comarcal y el bienestar de su gente. (Copus, 2015).

Incluso, como veremos, tienen a su disposición la imaginación para estimular otro tipo de conexión del mundo rural atrayendo, de diversas maneras, población diferente, flotante y temporal al espacio rural. Esta es, tal vez, la gran novedad que presenta nuestro trabajo. Junto a formas de vínculos con lo rural ya mencionadas anteriormente, existen otras que no están basadas en el efecto llamada de lo rural, sino que asumen que el espacio rural y urbano es fluido al igual que las identidades, y que la economía y las formas de vida lo son también, al modo como ha descrito Zygmunt Bauman (2007, 5). Esta forma de vínculo está muy lejos de ser nueva, es tan antigua como las peregrinaciones, y que la experiencia del camino de Santiago que recorre la comarca del Bierzo de este a oeste se ha encargado durante siglos de demostrar. En este caso el espacio rural, en determinados momentos aumenta significativamente de población, no es una población fija, no es residente, pero contribuye al conocimiento del lugar, a la riqueza y a la economía del mismo, a su expansión y a la ruptura de sus fronteras, así como también a la autoestima de sus habitantes.

"Hasta de Nueva Zelanda hemos visto peregrinos este año, y todos decían que les gustaba mucho esto. Nunca hubiéramos pensado que vinieran aquí desde tan lejos. Ellos se llevan un poquito del Bierzo a su país».

(Comentario de la propietaria de restaurante en el Acebo en el Camino de Santiago en el Bierzo).

Esta idea ha estado muy presente en el valle de Balboa desde hace algunos años. Es importante, como ellos mismos dicen, lo primero que no renunciemos a lo que somos. Esta es nuestra historia y nuestra identidad. Primero lo que somos. Luego que se conozca fuera lo que somos y lo que tenemos. Por eso es determinante el apoyo a los productos locales, a los productores locales y a la creación de empresas en el entorno rural que tengan mercados fuera de este entorno. Como veremos en la investigación etnográfica las nuevas tecnologías pueden contribuir 
CARLOS MONTES PÉREZ

COHESIÓN TERRITORIAL Y SOSTENIBILIDAD EN LOS VALLES DEL BIERZO

de un modo decisivo al crecimiento de estas nuevas empresas rurales. Y, por último, aunque no menos importante, es importante que la gente venga, aunque no se quede, aunque su estancia sea temporal. Y para ello es necesario usar la imaginación para crear vínculos que vayan más allá de una tarde, vínculos que se repitan y que consoliden una relación entre lo rural y lo urbano. El caso que veremos de la adopción de los sotos de castaños en el valle de Balboa que analizaremos posteriormente va en esta dirección y contribuye, por tanto, de un modo decisivo a la cohesión territorial.

\section{ESPACIOS RURALES FLUIDOS}

En el valle de Balboa han coincidido en los últimos años varios elementos que han ayudado a configurar un modelo de ruralidad resistente, no ha supuesto un aumento de la población, pero tampoco un descenso significativo en los últimos años. Cuandos los protagonistas reflexionan sobre su pasado, y sobre su quehacer en su entorno rural, señalan de forma reiterada la importancia de dos generaciones que, en su juventud decidieron resistir, imaginar mundos rurales posibles y ver posteriormente qué pasaba. La primera generación hace casi cuarenta años puso las bases para lo que hoy es el valle. Algunos de aquellos jóvenes que cuando se iniciaba la diáspora de los valles bercianos hacia otras latitudes decidieron quedarse se implicaron políticamente, tomaron la alcaldía y desde allí comenzaron un largo camino de mejora de las condiciones de la vida rural.

En los años 80 hay en Balboa un grupo de unos cien jóvenes que tienen una querencia por quedarse en el pueblo. Ya había pasado la etapa fuerte de la emigración y decidieron quedarse. Al principio fue como una rebeldía juvenil, lo que buscábamos era una discoteca para pasar el rato los fines de semana sin salir del valle, pero luego, ya nos fuimos dando cuenta que había que implicarse en política para cambiar algo. Al principio decidimos mejorar las infraestructuras esenciales, alcantillas, y calles, pero luego ya nos fuimos dando cuenta que algo más teníamos que hacer para que la gente que se quisiera quedar estuviera contenta de hacerlo.

(Monteserín, antiguo alcalde de Balboa).

Pero pasados unos años, esta generación se ha ido retirando de la vida pública y ha tomado el mando una nueva generación que toma como referencia los objetivos del mantenimiento de la población, y la mejora del bienestar de la vida de la gente del pueblo, pero ya en un contexto muy cambiante, de mundo más global y tecnológicamente mediado. En este sentido, la sostenibilidad ambiental y poblacional se ha basado en un conjunto de acciones llevadas a cabo por esta nueva generación de habitantes de la localidad de Balboa basadas sobre todo en la diversificación de propuestas, tal y como expondremos a continuación de forma breve. En este sentido se han llevado a cabo iniciativas de carácter institucional fruto de decisiones políticas que han tenido que priorizar unos objetivos en lugar 
de otros, y que han supuesto el enorme peso de la responsabilidad de haber decidido bien. Así se ha reconvertido un pabellón de deportes que se había quedado prácticamente sin uso, en la llamada Casa de las Gentes, lugar ya emblemático de la localidad que tiene un carácter de multiusos y que acoge, entre otras cosas un museo de la madera y salas de carácter polivalente para actividades diversas. Junto a esto se han puesto en marcha iniciativas como la adopción de castaños, creación de un albergue, mejora de distintas comunicaciones y la mejora de la situación de la localidad en relación a las tecnologías de la información. Estas iniciativas institucionales que describiremos con más detalle a continuación son importantes, si bien, no pueden soportar la tensión migratoria por si solas, si no van acompañadas de otras medidas y propuestas. Así se ha puesto de manifiesto en el valle de Balboa y puede servir como modelo para lugares diferentes con problemáticas similares.

Junto a lo comentado, desde el Ayuntamiento se ha promovido la recuperación de los motores económicos clásicos del valle y que han ido, poco a poco, desapareciendo. En concreto se ha recuperado de nuevo la actividad ganadera en el valle promoviendo de nuevo una feria de ganado al modo cómo estuvo presente ya en épocas pretéritas y se ha apoyado al cultivo de la castaña que había entrado en un periodo de letargo con la creación del Centro de Transformación de la Castaña, y la promoción de empresas enraizadas en el ámbito rural y que están relacionadas de una u otra manera con este fruto emblemático del valle.

Lo descrito hasta ahora ha supuesto un impulso desde los poderes públicos y con los recursos públicos, pero la sostenibilidad del medio rural necesita también la combinación con el impulso privado. En este sentido también ha tenido lugar un apoyo especial hacia aquellas iniciativas privadas que han apostado por recuperar aspectos de la arquitectura tradicional como han sido las pallozas y su dinamización como lugar de encuentro relacionado, sobre todo con la música. De tal modo que, actualmente la localidad de Balboa es conocida en todo el noroeste ibérico por sus festivales de música y sus encuentros de literatura y cenas concierto. Como veremos estas iniciativas han logrado consolidar antes de la pandemia un flujo constante de visitantes, que si bien, no han aumentado la residencia en la localidad, si, al menos han contribuido económicamente al sostenimiento y aprovechamiento de estas iniciativas. El caso del valle de Balboa es especialmente significativo en cuanto a la recepción de turistas y la capacidad de recibir flujos de visitantes, puesto que su número ha superado algunos años las 20.000 personas en una localidad que no supera los 400 habitantes.

En último lugar nos ocuparemos del eslabón final de esta compleja cadena como son las empresas rurales. La sostenibilidad rural, tal y como hemos puesto de manifiesto supone un ensamblaje de distintos elementos que combinen lo público y lo privado. En este sentido en el valle han surgido varias iniciativas empresariales dignas de mención de las que nos ocuparemos también en este trabajo. 


\section{DeCISIONES INSTITUCIONALES}

En los procesos de desarrollo rural tienen una importancia destacada pequeñas decisiones que pueden cambiar la dinámica económica y poblacional. En algunos casos pasan desapercibidas por falta de comunicación o bien por la creencia en su insignificancia, pero con el tiempo, se manifiestan como fundamentales. Tal ha sido el caso en la localidad de Balboa de transformar un pabellón deportivo en un centro distinto, la llamada casa de las gentes.

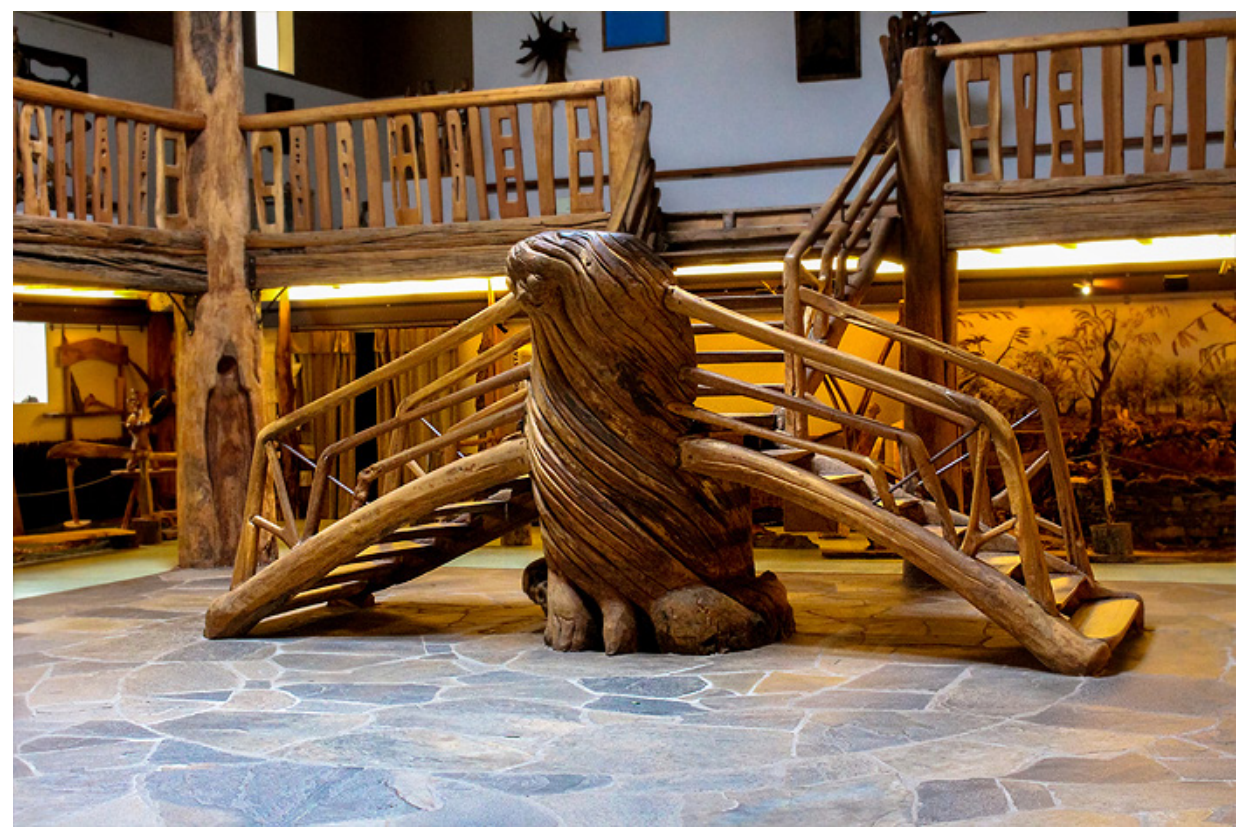

Figura n. ${ }^{\circ}$ 5. La casa de las Gentes. Balboa. Interior.

Este lugar ha contribuido de forma decisiva al mantenimiento de la población. Como comentamos el éxito radica en la visión de la transformación de un polideportivo para unos jóvenes que ya no hay en un museo que atesore lo mejor de lo que la tierra del valle ofrece, como es piedra, madera de castaño y la creatividad local. Gran parte del museo gira en torno al artista local Domingo González de Canteixeira, quien ha diseñado el puente que da acceso al museo, su interior y muchas de las piezas que el museo ofrece al visitante. La idea germinal consistía en ofrecer un espacio cultural distinto, donde el contenido y el continente llamaran la atención por su peculiaridad, y además, mostrar como puede haber una continuidad creativa entre la cultura tradicional representada por la madera, el puente de paso, el corredor y la decoración que ofrece una referencia 
a las pallozas con el hogar en el centro de la misma, entre otros elementos, y las formas artísticas que nacen de la creatividad individual del artista local, tal y como se percibe en las esculturas que el museo ofrece, entre las que sobresale la pareja tallada en un tronco único de castaño. Esto no ha sido la panacea para el valle, ciertamente nada lo es, pero sí ha sido un punto de interés para turistas y visitantes que han mostrado una grata sorpresa al encontrarse un museo tal en este lugar apartado de los núcleos más poblados. Este primer contacto ha hecho funcionar el boca a boca, y ha puesto a Balboa y a su Casa de las Gentes en el mapa turístico del Bierzo.

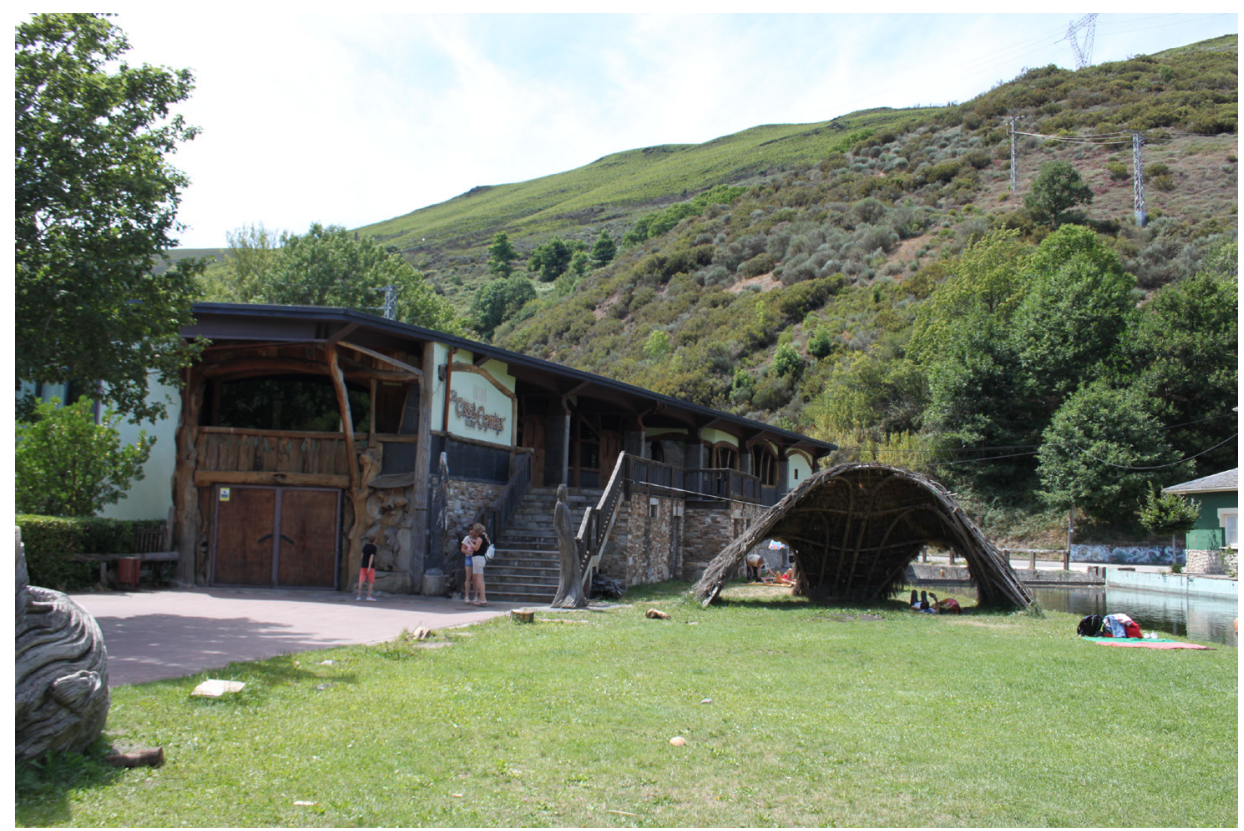

Figura n. ${ }^{\circ}$ 6. La Casa de las gentes. Exterior.

El contenido cambia con el tiempo, en este lugar han tenido lugar exposiciones variadas, ciclos de conciertos y conferencias, y ha sido también lugar de reunión de productores locales, así como de gentes diversas del valle para distintas iniciativas. Ha sido, y lo sigue siendo un lugar de cultura y un lugar de reunión, un espacio, como al exalcalde Monteserín le gusta decir, un lugar para la gente de aquí y de allí, y de cualquier otro sitio.

Otra iniciativa institucional que ha contribuido al conocimiento del valle, a su sostenibilidad y a su flujo constante de visitantes es el programa de adopción de castaños puesto en marcha desde el Ayuntamiento. Este programa ha supuesto un soplo de aire fresco para la localidad. Este proyecto se gestó también entre la 
corporación municipal encabezada por el antiguo alcalde, verdadero promotor y defensor del valle. El programa nace en un contexto de bajos precios de la castaña "de parede» y el posterior abandono de aquellos sotos de castaños que no producen un buen rendimiento. Este deterioro ha supuesto un alto peligro de incendios en los veranos por una falta de cuidado en los caminos de acceso y entre los castaños. El programa "adopta un castaño" tuvo en enorme éxito. Se inició en el año 2010 con la puesta en marcha de varios anuncios en medios de comunicación locales y con la recepción en la Casa de las Gentes de un buen número de familias que acudieron a la llamada de Balboa. La idea era sencilla, pero interesante desde el punto de vista del desarrollo local y de la sostenibilidad tanto ambiental como económica. Se trataba también, en definitiva de conectar, de hacer realidad el continuum rural y urbano a través de la adopción de varios castaños. En la primera convocatoria fueron casi cien participantes los que acudieron a la llamada, de varios puntos de España. La mayoría de la provincia de León y de la capital, pero también de varias zonas de Galicia, de Asturias y de Valladolid. En esta primera convocatoria se fueron asignando los castaños que estaban ya numerados de un soto en dirección a Cantexeira cuyo acceso estaba limpio y preparado por los trabajadores del Ayuntamiento. Cada familia adoptante asumía unos compromisos con sus castaños adoptados y tenía también unos derechos que tenían que ver con la recolección y la recogida del fruto en el otoño. Mayoritariamente acudieron a la llamada familias con niños pequeños que de forma regular debían acudir a la localidad de Balboa al menos dos veces al año para cumplir con la obligación de la limpieza del soto, y luego acudirían una o dos veces más para la recolección de las castañas. De este modo, el programa pretendía cumplir varios objetivos, el primero de ellos era el mantener los sotos de castaños limpios y protegidos, en segundo lugar establecer un vínculo rural-urbano, de modo que las familias que acudían a la localidad se convertían en potenciales consumidores de los productos y de los negocios locales, y en tercer lugar y muy importante, se dotaba de visibilidad al valle. El boca a boca de los adoptantes en esta primera convocatoria fue grande, lo que posibilitó una segunda convocatoria en otro soto distinto. El programa, debido a la subida del precio del fruto en años venideros, y las distintas crisis económicas fue, poco a poco, mermando hasta su extinción, pero ha quedado como idea generadora de desarrollo en un entorno sostenible, tal y como se puso de manifiesto en el blog que se creó a tal efecto.

Te ofrecemos la posibilidad de adoptar un castaño. Podrás venir a recoger el fruto de tu árbol asignado. Solo tienes que comprometerte a realizar tareas de limpieza y mantenimiento una vez al menos a lo largo del añoDesde el Ayuntamiento se ha puesto en marcha una iniciativa que pretende rescatar del abandono los sotos de castaños. Cómo muchos ya sabéis, en el valle abundan los de la variedad Castaña de Parede. Esta castaña, sabrosísima y fácil de pelar, tiene el relativo defecto de ser pequeña y algo más arrugada (sin duda concentra más el sabor), aspectos por los que no es valorada como se merece en el sagrado mercado y su precio se mantiene en mínimos desde hace muchos años. 
CARLOS MONTES PÉREZ

COHESIÓN TERRITORIAL Y SOSTENIBILIDAD EN LOS VALLES DEL BIERZO

El abandono es hasta cierto punto lógico cuando el rendimiento es tan mínimo, por lo que sólo la población más mayor, y cada vez menos, las sigue recogiendo como un acto tradicional. Poder recogerlas en las mejores condiciones supone haber desbrozado y limpiado y éso se hace previamente varias veces al añoEl Ayuntamiento se está poniendo en contacto con propietarios que por una razón u otra no los mantienen en las debidas condiciones para gestionar los sotos y ponerlos en valor. El propietario cede el uso y gestión del soto, sin contraprestación económica. Un primer acuerdo con varios propietarios ha posibilitado el primer Banco de Castaños de Balboa, que cuenta en la actualidad con más de 7 hectáreas de sotos conteniendo más de 250 castaños.

(extracto del Blog «adopta un castaño», 2010).

De este programa ha quedado, sin duda, la idea y la experiencia de crear una nueva idea de ruralidad flexible, lábil, basada, no en territorialidades permanentes, sino más bien, en un modo de pensar el espacio rural de un modo líquido que pueda sostener y mantener la población a través de flujos. Por este motivo, esta categoría, tan habitual en los análisis de la antropología urbana puede ser aplicado también al desarrollo rural. (Appadurai, 2001) (Bauman, 2012). Estas iniciativas van sumando poco a poco. Ninguna de ellas por si sola es capaz de revertir una complicada y compleja situación territorial, pero el conjunto de muchas de ellas logra algún efecto. Unida a esta idea de adoptar un castaño, hay que mencionar también la propuesta de creación del primer carril-vaca de Europa. Así fue como fue recogida la idea en los principales medios de comunicación comarcales y provinciales hasta dar el salto a algunos medios nacionales. En esta caso, y tomando como referencia el llamado carril bici, la corporación municipal propuso unir los dos recursos principales del valle como son el turismo, por un lado y la ganadería por otro. Así, al tiempo que se arreglaba la infraestructura viaria principal de acceso a la localidad desde Ambasmestas, se habilitaba también un carril para que el ganado pudiera circular en busca de pastos. Este guiño pretendía de nuevo visibilizar el valle, colocarlo en los medios de comunicación y resaltar la importancia que la ganadería tiene aún en el valle, a diferencia de otros rincones del bierzo donde prácticamente ha desaparecido.

\section{DECISIONES EMPRESARIALES}

Tal y como hemos puesto de manifiesto las iniciativas institucionales son fundamentales en todo proceso de desarrollo rural, si bien, para su consolidación son necesarias también otro tipo de decisiones de tipo particular y que tengan una dimensión empresarial. Este debe de ser uno de los objetivos fundamentales de todo proceso sostenible de mejora de los territorios rurales. Así hemos podido constatar y documentar etnográficamente el surgimiento de varias empresas en la localidad de Balboa que apuestan, en principio por aprovechar estos flujos de visitantes que, entre quincemil a veintemil visitantes al año, y las nuevas tecnologías 
que se han convertido en el verdadero reto de los espacios rurales, sobre todo de montaña. Así, en este contexto es como nace la empresa rural La oricera, empresa familiar destinada a la elaboración de forma artesanal de productos derivados de la castaña. Esta empresa asentada en Balboa compra el producto a las productores locales de esta modalidad de castaña que resulta pequeña y con una salida escasa en el mercado para llevar a cabo con ella distintos productos de elaboración y transformación.

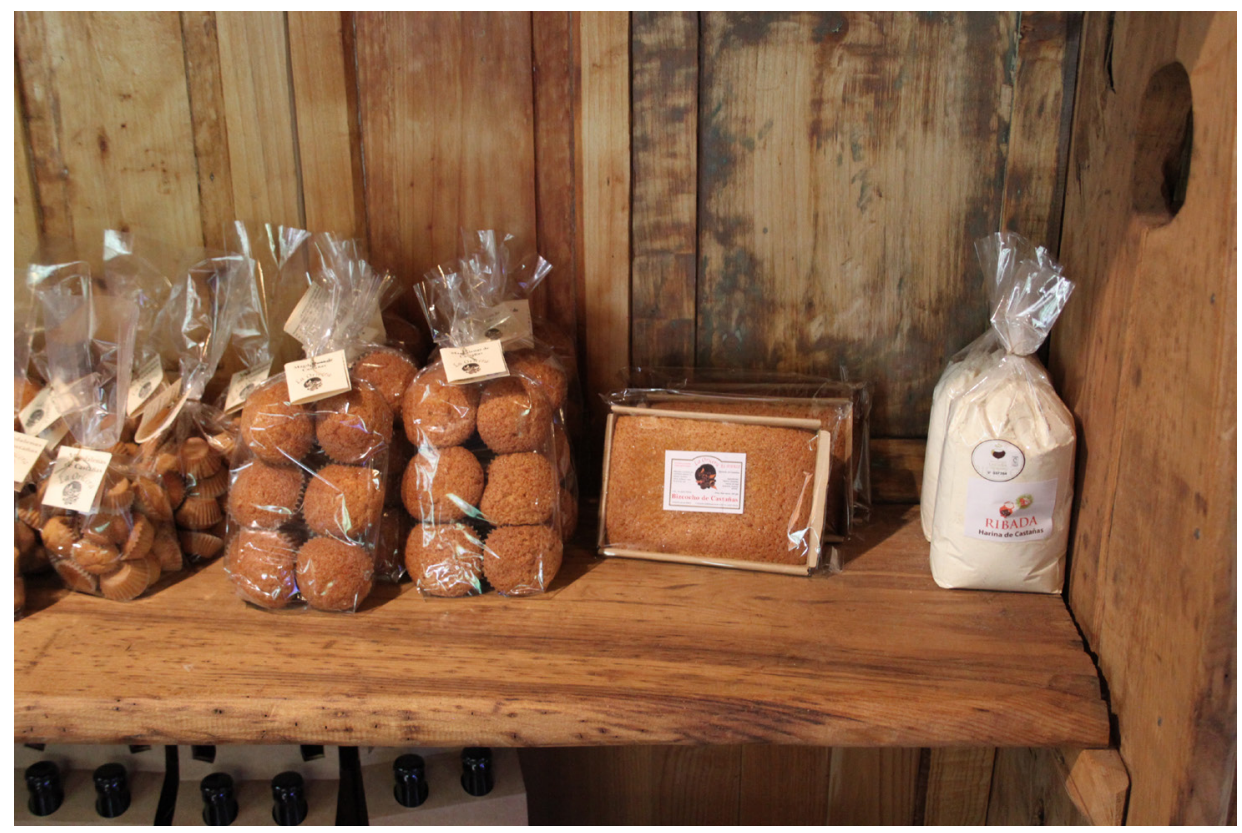

Figura n. ${ }^{\circ}$. Productos elaborados con harina de castaña.

El éxito de la empresa consiste, sobre todo, en haber tomado el producto más característico de la zona, haber implicado en el proyecto a los productores locales, y haber apostado por la transformación de un producto que tradicionalmente se ha exportado en el Bierzo. La empresa ha diversificado ya los productos que elabora, destaca la harina de castaña, y la repostería realizada con dicha harina, como son lejas magdalenas, los bizcochos y las tartas, pero lo que destaca por encima de cualquier otro producto actualmente es la cerveza de castaña. La empresa tiene también un bar para poder degustar los productos y para dar a conocer los mismos, así como los encantos del valle.

Esta iniciativa empresarial ha mejorado las expectativas de varios productores de castañas que durante algún tiempo dejaban incluso de recolectar por la falta de precio y de salida para el producto y ha sido uno de los motivos de la cancelación 
del proyecto de adopción de castaños. Debido a estas iniciativas y a otras similares en la zona los sotos de castaños comenzaron de nuevo a resultar rentables, y los propietarios que los cedieron al Ayuntamiento y aquellos que los abandonaron vieron de nuevo un recurso económico que merecía la pena conservar y explotar.

La presencia de La Oricera en internet y en redes sociales, así como su presencia en mercados locales y comarcales ha contribuído de forma decisiva al mantenimiento del proyecto, han aumentado sus ventas y sus cervezas, sobre todo, ya son conocidas y comercializadas con éxito en la comarca y en la región.

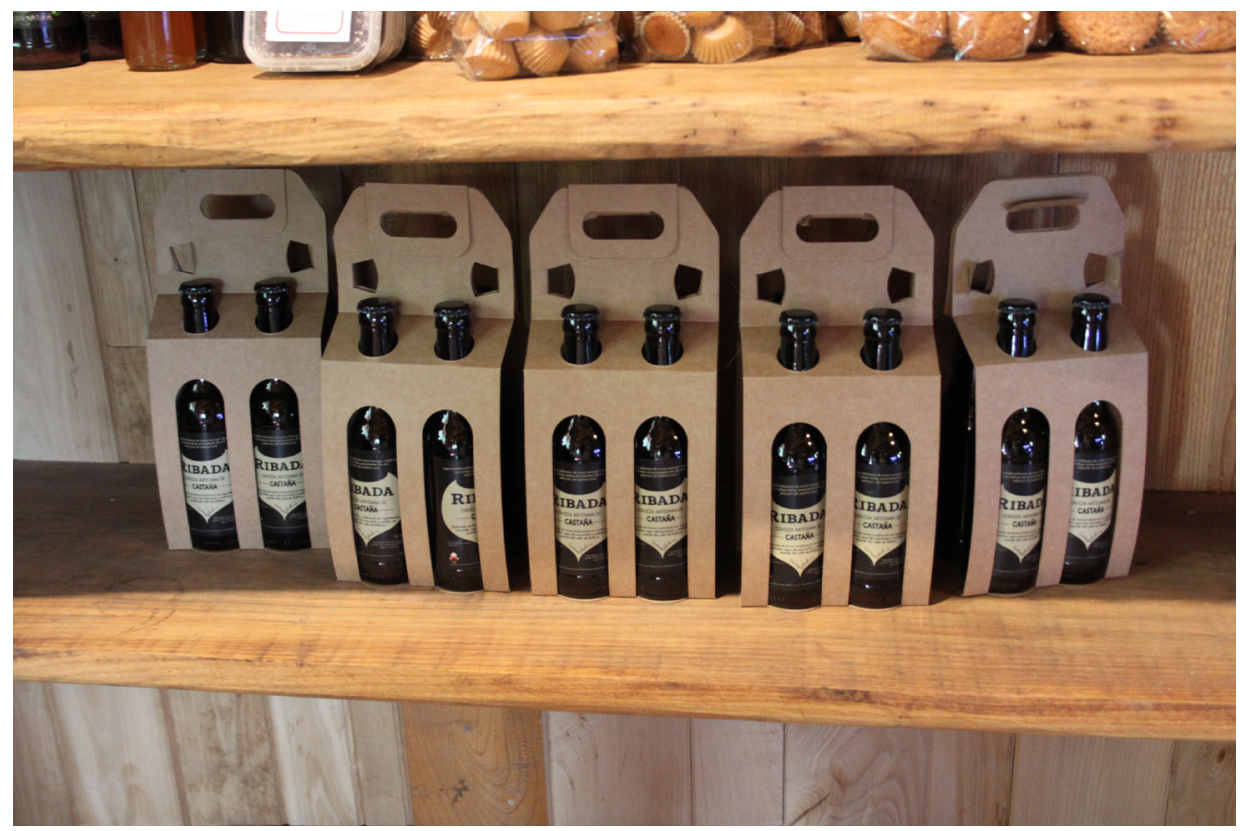

Figura n. ${ }^{\circ}$. Cerveza artesana de castaña.

\section{A MODO DE CONCLUSIÓN}

El espacio rural de montaña, y periférico se encuentra sometido a una grave y delicada tensión poblacional que conlleva deficiencias desde el punto de vista de los servicios, infraestructuras, mercados y desarrollo económico y vital. Las organizaciones de distinta índole y las instituciones son verdaderamente conscientes del problema y, a pesar de haber invertido mucha energía y dinero, el problema de la despoblación en determinados espacios rurales no ha mejorado. Por tal motivo es importante abordar el problema desde una doble perspectiva. En principio, buscando modelos que tengan algo significativo que ofrecer en torno al desarrollo y 
la sostenibilidad rural, y en segundo lugar, y a partir de los ejemplos mostrados, configurar un paradigma distinto que nos permita entender la sostenibilidad en espacios rurales de otro modo, sin enfatizar especialmente el enraizamiento y la territorialidad, sino con ideas más abiertas que reconfiguren la presencia de lo rural a través de la idea de flujo, de temporalidad, o de ruralidad líquida. (Gómez Pellón, 2012).

Por tal motivo nos ha parecido de especial importancia el caso del valle de Balboa como ejemplo de resistencia rural y de buenas y creativas prácticas destinadas a superar esta tensión migratoria que, en muchos lugares se acaba imponiendo con el tiempo. En el caso que hemos presentado se unen iniciativas de tipo público e institucional que, sin ser ninguna solución definitiva son fundamentales para el fermeto de otras iniciativas y decisiones de tipo particular que constituyen el objetivo a largo plazo. El caso es significativo además por haber puesto de manifiesto cómo la idea de flujo temporal de visitantes puede ser también un modelo de desarrollo rural. En esta localidad está siendo especialmente relevante la ruptura de la barrera entre lo rural y lo urbano, a través de presencias temporales, pero numerosos y continuadas de distintos flujos de visitantes en el valle. No deja de sorprender cómo una localidad que no reúne a más de trescientos vecinos en época estival, y muchos menos en el invierno, pueda contar anualmente con más de quince mil visitantes gracias a sus programas de adopción de castaños, de festivales celtas, de atracciones turísticas, de gastronomía de pallozas, de tertulias literarias, de cenas temáticas y de magostos celtas, entre otras iniciativas. Ahora bien, lo que resulta claro de esta realidad es que detrás hay voluntad de desarrollo y de no bajar los brazos ni resignarse, y, sobre todo, hay una buena cantidad de creatividad y de amor por un territorio que resulta embaucador y digno de admiración y de disfrute. Parece claro por tanto, como conclusión a lo expuesto que cada elemento que configura el mundo rural es importante en los procesos de desarrollo Los administradores y grupos de gobierno tienen a su disposición más o menos recursos que han de ser priorizados de un modo racional, inteligente y creativo. Los fondos pueden ser usados para la construcción de un pabellón de deportes en una población envejecida o bien, para la creación de la Casa de las Gentes, y la aportación al desarrollo local será distinta. También se perciben diferencias en el modo de abordar las iniciativas propuestas. No todos los pueblos de montaña han ideado programas de adopción de castaños o reivindicación de un carril-vaca. La creatividad fomenta la visibilidad y esto a través de las redes sociales es un elemento destacado para poder iniciativas de distinta índole. En caso de Balboa hay algunos otros elementos que han desempeñado un papel importante, pero que no han podido ser abordados en este trabajo, como es su condición límite entre Castilla, Galicia y Asturias y la reivindicación de una identidad y un pasado unido a la cultura castrense y celta del noroeste ibérico. Este aspecto le ha permitido organizar festivales temáticos con gran afluencia de jóvenes en las primaveras y veranos con la aquiescencia y beneplácito de los más ancianos de la localidad, como si de un nuevo e implícito contrato social se tratase. 
En definitiva, buena gestión de los recursos y creatividad para competir en un mundo globalizado, generación de infraestructuras por parte de los poderes públicos y apoyo a las iniciativas privadas que nazcan del entorno cercano y potencien los productos locales son elementos, que, sin asegurar una significativa mejoría, si, al menos, permite luchar contra el olvido y el abandono.

\section{REFERENCIAS BIBLIOGRÁFICAS}

Alonso Santos, J. L. (2003). Redes y procesos de innovación en las comarcas vinícolas del Castilla y León: el ejemplo de la D.O. Bierzo. Boletín de la asociación de geógrafos españoles, 36, pp. 43-60.

Amselle, J. (2010). Le retour de l'indigène. L'homme, revue française d'anthropologie, París, 194, pp. 131-138.

Appadurai, A. (2001). La modernidad desbordada: dimensiones culturales de la globalización. Buenos Aires: Ediciones Trilce, Fondo de Cultura Económica.

Bauman, Z. (2007). Tiempos líquidos: vivir en una época de incertidumbre. Barcelona: Tusquets.

Bauman, Z. (2012). Modernidad líquida. Buenos Aires: Fondo de Cultura Económica.

CCE. (comisión de las comunidades europeas). (2008). Libro verde sobre la cohesión territorial: convertir la diversidad territorial en punto fuerte. Bruselas (6-10-2008).

Copus, A. K., De Lima, P. (edit.) (2015). Territorial Cohesion in rural Europe: The relational turn in rural development. New York, Routledge.

Cortizo, J., Maya, A. (1992). La actividad agraria en el Bierzo: la comarca del Bierzo. Ponferrada, 7, pp. 21-39.

Esparcia, J. (2004). Políticas públicas en el medio rural: desarrollo rural: contribución de los programas leader y proder al desarrollo rural en España. F. Molinero, R. Majoral, J. M. Bartolomé, G. García Fernández, (coords.), Atlas de la España rural, (pp. 380-384). Madrid: Centro de publicaciones del Ministerio de Agricultura, Pesca y Alimentación.

Gómez Pellón, E. (2012). Ruralidad y discurso: del caso español al de Cantabria. AIBR: Revista de antropología iberoamericana, 7(3), pp. 295-326.

González Vecín, J. (1984). Diferencias espaciales en la agricultura de el Bierzo, León. León: Publicaciones Universidad de León.

Zafra, R. (2010). Un cuarto propio conectado. Madrid: Fórfola. 